\title{
Clinical Course After Initiation of Nivolumab Therapy in Patients with EGFR-Mutated Non-Small Cell Lung Cancer With or Without Pd-L1 Expression
}

\author{
Minehiko Inomata $(\mathbb{D} \cdot$ Hiroaki Tanaka $\cdot$ Kotaro Tokui $\cdot$ \\ Chihiro Taka · Seisuke Okazawa - Kenta Kambara · Shingo Imanishi · \\ Toru Yamada · Toshiro Miwa · Ryuji Hayashi · Kazuyuki Tobe
}

Received: August 23, 2017 / Published online: October 20, 2017

(C) The Author(s) 2017. This article is an open access publication

\section{ABSTRACT}

Introduction: Nivolumab has been reported to be more effective in the subset of non-squamous non-small cell lung cancer (NSCLC) patients with tumors harboring wild-type epidermal growth factor receptor (EGFR) and/or expressing programmed death ligand-1 (PD-L1). However, it is unclear whether PD-L1 expression influences the efficacy of nivolumab in patients with EGFR-mutated NSCLC. Therefore, tumor PD-L1 expression was evaluated in five patients with EGFR-mutated NSCLC who had been treated with nivolumab.

Methods: We have identified patients with NSCLC harboring EGFR gene mutation retrospectively out of a group of 23 consecutive patients treated with nivolumab at our hospital between January 2016 and March 2017. The tumors of patients with EGFR-mutated NSCLC

Enhanced content To view enhanced content for this article go to http://www.medengine.com/Redeem/ 98CCF06024DE3FD2.

M. Inomata $(\varangle) \cdot$ H. Tanaka $\cdot$ K. Tokui $\cdot$ C. Taka

S. Okazawa · K. Kambara · S. Imanishi ·

T. Yamada $\cdot$ T. Miwa $\cdot$ K. Tobe

First Department of Internal Medicine, Toyama University Hospital, Sugitani, 2630, Toyama, Japan

e-mail:minomata@med.u-toyama.ac.jp

R. Hayashi

Department of Medical Oncology, Toyama

University Hospital, Sugitani, 2630, Toyama, Japan who had received nivolumab treatment based on clinical judgment were analyzed retrospectively. Tumor PD-L1 expression was evaluated by measurement of the tumor proportion score (TPS) using a rabbit antihuman PD-L1 antibody (clone 22C3).

Results: Three patients received the treatment with nivolumab as second line treatment, and two patients received the treatment as third line treatment. One out of five patients had a response with treatment with nivolumab and that was the only patient with a positive PD-L1 expression (TPS of 80-90\%).

Conclusion: The results of the present study suggest that evaluation of tumor PD-L1 expression might be useful for predicting the efficacy of nivolumab, even in patients with EGFR-mutated NSCLC.

Keywords: Epidermal growth factor receptor; Immune check point inhibitor; Non-small cell lung cancer; Programmed death ligand

\section{INTRODUCTION}

Recently, nivolumab, fully human IgG4 programmed death-1 (PD-1) immune-checkpoint-inhibitor antibody, was demonstrated to confer survival benefit as compared to docetaxel, the standard chemotherapy for previously treated NSCLC patients, in two 
randomized controlled trials, Check Mate 017 and Check Mate $057[1,2]$.

The results of the Check Mate 057 trial suggested that nivolumab was more effective in the subset of non-squamous NSCLC patients with tumors harboring wild-type epidermal growth factor receptor (EGFR) and expressing programmed death ligand-1 (PD-L1) [2]. In addition, the Key Note 010 trial, in which the efficacy of another immune check point inhibitor, pembrolizumab, was investigated, also yielded similar findings [3].

The current level of evidence supports the use of checkpoint inhibitors in NSCLC patients in both first line and subsequent lines. The chance of having a benefit with these increases with the level of PD-L1 expression, and it seems to decrease if EGFR mutations are present. However, there is still insufficient evidence on how PD-L1 expression influences the efficacy of immune checkpoint inhibitors in patients with EGFR-mutated NSCLC. The present study was aimed at evaluating the impact of tumor PD-L1 expression on the efficacy of immune checkpoint inhibitors in patients with NSCLC harboring EGFR gene mutations.

\section{METHODS}

This observational study was conducted retrospectively. Patients with EGFR-mutated NSCLC who had been treated with nivolumab based on clinical judgment were included. We reviewed the patient data, including the age, gender, performance status, smoking status, progression-free survival (PFS) from the initiation of treatment, and response to nivolumab from the medical records.

Immunohistochemistry to determine tumor PD-L1 expression was commissioned to a commercial laboratory (BML Inc., Tokyo). PD-L1 expression was evaluated by the tumor proportion score (TPS), which refers to the proportion of tumor cells expressing PD-L1 in tumor biopsy specimens obtained from the patients, determined using a rabbit antihuman PD-L1 antibody (clone 22C3).

The present study was conducted with the approval of the Ethics Committee of the
University of Toyama (Reference Number: 29-10), by taking the informed consent for the publication for this study and any additional related information from the patients involved in the study and/or disclosing the information about study plan, according to Ethical Guidelines for Medical and Health Research Involving Human Subjects (Ministry of Health, Labor and Welfare of Japan).

\section{RESULTS}

All of 23 patients with NSCLC had received treatment with nivolumab in Toyama University Hospital between January 2016 and March 2017. Of these, five patients showed EGFR activating mutations, including exon 19 deletion and exon 21 L858R point mutation, in the tumor.

Table 1 shows the patient characteristics and response to nivolumab treatment. All patients with EGFR-mutated NSCLC had received the treatment with EGFR-TKI at any point of the clinical course. Patients number 1,3 , and 4 received the treatment with nivolumab as second line treatment, and patients number 2 and 5 received the treatment as third line treatment. Patients number 1, 2, and 5 received a biopsy immediately before the treatment with nivolumab. In patient number 4 , a tissue specimen that was obtained at initial diagnosis was used for immunohistochemistry. In patient number 3 , initial diagnosis and biopsy was conducted in another hospital, and the specimen could not be used for the present study. The patient received a biopsy after the nivolumab therapy for the clinical necessity, and the specimen was used for immunohistochemistry. Four patients showed disease progression after the initiation of treatment with nivolumab. Pseudo-progression was excluded in each patient based on clinical judgement including concomitant deterioration of symptoms and performance status, or findings of a computed tomography scan conducted afterward. The PFS in these patients ranged from 0.6 to 4.2 months, and no response to nivolumab was observed. Immunohistochemistry revealed that the TPS in the tumor specimens obtained from these 
Table 1 Patient characteristics and response to nivolumab

\begin{tabular}{lllllllll}
\hline & Gender & Age & Pack-year & Biopsy site & $\begin{array}{l}\text { Prior } \\
\text { treatment }\end{array}$ & TPS & PFS $^{*}$ & Response \\
\hline 1 & Female & 87 & 0 & Lung & EGFR-TKI & $<1 \%$ & 1.0 & PD \\
2 & Female & 64 & 0 & Liver & EGFR-TKI & $<1 \%$ & 1.0 & PD \\
3 & Female & 68 & 0 & Bone & Cytotoxic agent & $<1 \%$ & 4.2 & Non-CR/ \\
& & & & & & & & non-PD \\
4 & Male & 65 & 40 & Lung & EGFR-TKI & $<1 \%$ & 0.6 & PD \\
5 & Male & 68 & 75 & Liver & Cytotoxic agent & $80-90 \%$ & $7.9^{\dagger}$ & PR \\
\hline
\end{tabular}

$C R$ complete response, $P D$ progressive disease, $P R$ partial response, $T P S$ tumor proportion score

${ }^{*}$ Months

${ }^{\dagger}$ Censored

patients was less than $1 \%$. In patient number 5 , the mediastinal lymph node metastasis responded to nivolumab, corresponding to "partial response" according to the Response Evaluation Criteria in Solid Tumors. Immunohistochemistry of the tumor specimen obtained from this patient revealed a TPS of $80-90 \%$. The patient experienced appetite loss of grade 3 requiring admission and elevation of serum creatinine level of grade 2 . These adverse events were improved after the cessation of nivolumab. However, appetite loss and pneumothorax in the left lung were observed after the re-administration of nivolumab. Although disease progression was not detected by clinical testing including computed tomography imaging, treatment with nivolumab was ceased because of appetite loss and poor performance status. The patient was referred to another hospital at 7.9 months later after the initiation of the treatment with nivolumab.

\section{DISCUSSION}

Although tumor PD-L1 expression has been reported to be associated with better treatment efficacy of nivolumab in patients with non-squamous NSCLC [2], the response rate to nivolumab in patients with EGFR-mutated NSCLC is known to be low [4]. Therefore, there is the clinical question of which treatment option can be recommended for patients with EGFR-mutated NSCLC showing PD-L1 expression. The present study showed that the efficacy of nivolumab was correlated with the expression status of PD-L1, even in patients with EGFR-mutated NSCLC.

EGFR activation by EGF stimulation is reported to upregulate PD-L1, and it has also been demonstrated that inhibition of EGFR by EGFR-TKI downregulated PD-L1 [7, 8]. Given these previous reports, the association is expected between PD-L1 expression and EGFR gene mutation. However, there are inconsistent reports from previous clinical studies on the association between PD-L1 expression and EGFR gene mutation [4-7]. PD-L1 expression could be regulated by several factors including oncogene pathway activation and cytokines such as interferon $\gamma$, and EGF signals might be one of the underlying mechanisms for expression of PD-L1 in NSCLC harboring EGFR gene mutation [8].

There are several limitations in the present study. The first is a very small sample size. Although the concordance of PD-L1 expression with efficacy of nivolumab was shown in five patients, it might be observed by chance, and we cannot draw a definitive conclusion. So, a study with a larger population is needed for verification. Second, in patient number 3, because the specimen obtained after the treatment with nivolumab was used for immunohistochemistry, it cannot be excluded that PD-L1 status shown in the present study might not reflect the status before the treatment with nivolumab. 
In conclusion, the present study suggests an association between the PD-L1 expression status and the efficacy of nivolumab in patients with EGFR-mutated NSCLC. Evaluation for PD-L1 expression may be useful for predicting the efficacy of nivolumab and selection in patients with EGFR-mutated NSCLC.

\section{ACKNOWLEDGEMENTS}

No funding or sponsorship was received for this study or publication of this article.

All named authors meet the International Committee of Medical Journal Editors (ICMJE) criteria for authorship for this manuscript, take responsibility for the integrity of the work as a whole, and have given final approval to the version to be published.

Disclosures. Minehiko Inomata has received research grants from Daiichi Sankyo Co. Kazuyuki Tobe has received research grants from Fuji Chemical Industries Co., Ono Pharmaceutical Co., Kyowa Hakko Kirin Co., and Takeda Pharmaceutical Co. Hiroaki Tanaka, Kotaro Tokui, Chihiro Taka, Seisuke Okazawa, Kenta Kambara, Shingo Imanishi, Toru Yamada, Toshiro Miwa, and Ryuji Hayashi have nothing to disclose.

Compliance with Ethics Guidelines. All procedures performed in studies involving human participants were in accordance with the ethical standards of the institutional research committee (Ethics Committee of the University of Toyama, Reference Number: 29-10) and with the 1964 Helsinki Declaration and its later amendments or comparable ethical standards.

The present study was conducted by disclosing the information about the study plan and/or obtaining the informed consent, according to Ethical Guidelines for Medical and Health Research Involving Human Subjects (Ministry of Health, Labor and Welfare of Japan).
Data Availability. Data sharing is not applicable to this article as no datasets were generated or analyzed during the current study.

Open Access. This article is distributed under the terms of the Creative Commons Attribution-NonCommercial 4.0 International License (http://creativecommons.org/licenses/ by-nc/4.0/), which permits any noncommercial use, distribution, and reproduction in any medium, provided you give appropriate credit to the original author(s) and the source, provide a link to the Creative Commons license, and indicate if changes were made.

\section{REFERENCES}

1. Brahmer J, Reckamp KL, Baas P, et al. Nivolumab versus docetaxel in advanced squamous-cell non-small-cell lung cancer. N Engl J Med. 2015;373:123-35.

2. Borghaei H, Paz-Ares L, Horn L, et al. Nivolumab versus docetaxel in advanced nonsquamous non-small-cell lung cancer. N Engl J Med. 2015;373:1627-39.

3. Herbst RS, Baas P, Kim DW, et al. Pembrolizumab versus docetaxel for previously treated, PD-L1-positive, advanced non-small-cell lung cancer (KEYNOTE-010): a randomised controlled trial. Lancet. 2016;387:1540-50.

4. Gainor JF, Shaw AT, Sequist LV, et al. EGFR mutations and ALK rearrangements are associated with low response rates to PD-1 pathway blockade in non-small cell lung cancer: a retrospective analysis. Clin Cancer Res. 2016;22:4585-93.

5. Li D, Zhu X, Wang H, Li N. Association between PD-L1 expression and driven gene status in NSCLC: A meta-analysis. Eur J Surg Oncol. 2017. doi:10.1016/j. ejso.2017.02.008.

6. Rangachari D, VanderLaan PA, Shea M, et al. Correlation between classic driver oncogene mutations in EGFR, ALK, or ROS1 and 22C3-PD-L1 $\geq 50 \%$ expression in lung adenocarcinoma. J Thorac Oncol. 2017;12:878-83.

7. Okita R, Maeda A, Shimizu K, Nojima Y, Saisho S, Nakata M. PD-L1 overexpression is partially regulated by EGFR/HER2 signaling and associated with poor prognosis in patients with non-small-cell lung cancer. Cancer Immunol Immunother. 2017. doi:10.1007/ s00262-017-1986-y. 
8. Chen N, Fang W, Zhan J, et al. Upregulation of PD-L1 by EGFR activation mediates the immune escape in EGFR-driven NSCLC: implication for optional immune targeted therapy for NSCLC patients with EGFR mutation. J Thorac Oncol. 2015;10:910-23. 